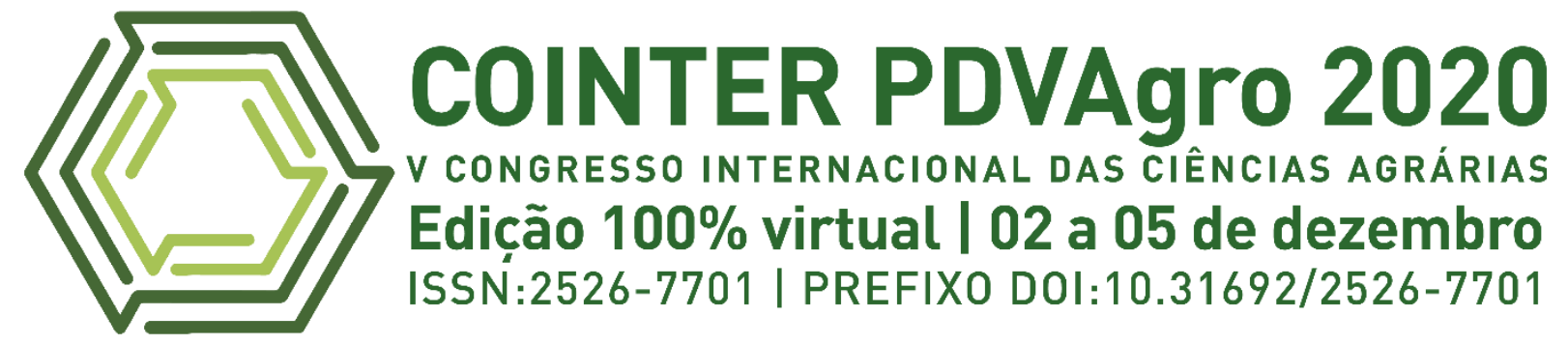

\title{
AVALIAÇÃO DE BIOSSEGURIDADE NA ESTAÇÃO DE AQUICULTURA DA ESCOLA AGRÍCOLA DE JUNDIAÍ
}

\section{EVALUACIÓN DE BIOSEGURIDAD EN LA ESTACIÓN DE ACUICULTURA DE LA ESCUELA AGRÍCOLA JUNDIAÍ}

\section{EVALUATION OF BIOSECURITY AT THE AQUACULTURE STATION OF THE JUNDIAÍ AGRICULTURAL SCHOOL}

\author{
Apresentação: Comunicação Oral \\ Gabriel Joaquim Santos Silva; Laércio Medeiros; Matheus Melo Dantas; Viviane da Silva \\ Medeiros.
}

\section{RESUMO}

DOI: https://doi.org/10.31692/2526-7701.VCOINTERPDVAgro.0241

A produção animal pode sofrer grandes impactos por conta das enfermidades que acometem a mesma. Dessa forma, para se evitar a entrada de patógenos nos sistemas de produção, o conceito de biosseguridade deve ser compreendido e aplicado de forma contínua em todos os segmentos produtivos. Este trabalho teve como objetivo avaliar as áreas de risco para entrada de patógenos nos setores da estação de aquicultura da Escola Agrícola de Jundiaí. Inicialmente, foi aplicado aos funcionários do local um questionário estruturado com perguntas abertas sobre alguns aspectos funcionais das atividades desempenhadas por lá. As suas respectivas respostas foram armazenadas a fim de se realizar uma avaliação qualitativa, dando sequência ao trabalho de análise e classificação das práticas de manejo vistas como fundamentais para a manutenção da biosseguridade. Desse modo, constatou-se algumas inadequações estruturais e técnicas que poderiam vir a acometer pontos vitais da segurança biológica do local. O artigo chama atenção para essas falhas e promove uma discussão em relação ao protocolo de biosseguridade dentro da aquicultura, abrindo margem para o aprimoramento produtivo tendo como base referencial o mesmo modelo que é desenvolvido em outras aéreas como suinocultura e avicultura. Desse modo, é garantido a Escola Agrícola de Jundiaí, unidade acadêmica especializada em ciências agrárias, um trabalho de aplicação atemporal constituído por preceitos que poderão servir de modelo para qualquer atividade que envolva a relação direta entre animais e humanos, garantindo maior estabilidade para que os resultados almejados sejam alcançados sem a incidência de agentes etiológicos e de outros fatores externos durante o processo.

Palavras-chave: Biosseguridade; Aquicultura; Controle de doenças; Agentes biológicos

\footnotetext{
1 Graduando em Química do Petróleo, Universidade Federal do Rio Grande do Norte - UFRN, email: gabrieljoaquim3@gmail.com

${ }^{2}$ Pós-Graduando em Tecnologias aplicadas a educação, Instituto Federal do Rio Grande do Norte - IFRN, email: laerciomedeiros2015@gmail.com

${ }^{3}$ Graduando em Medicina Veterinária, Universidade Federal da Paraíba - UFPB, email: melodantasm@ gmail.com

${ }^{4}$ Doutora, Escola Agrícola de Jundiaí - UFRN, email: vivianemedeiros.eaj@gmail.com
} 


\title{
AVALIAÇÃO DE BIOSSEGURIDADE NA ESTAÇÃO DE AQUICULTURA
}

\section{RESUMEN}

La producción animal puede sufrir grandes impactos debido a las enfermedades que la afectan. Así, para evitar la entrada de patógenos en los sistemas de producción, el concepto de bioseguridad debe ser entendido y aplicado de manera continua en todos los segmentos productivos. Este trabajo tuvo como objetivo evaluar las áreas de riesgo de patógenos que ingresan a los sectores de la estación acuícola de la Escuela Agrícola de Jundiaí. Inicialmente, se aplicó a los empleados del lugar un cuestionario estructurado con preguntas abiertas sobre algunos aspectos funcionales de las actividades que allí se desarrollan. Sus respectivas respuestas fueron almacenadas con el fin de realizar una evaluación cualitativa, continuando el trabajo de análisis y clasificación de las prácticas de manejo consideradas fundamentales para el mantenimiento de la bioseguridad. Así, se encontraron algunas deficiencias estructurales y técnicas que podrían afectar puntos vitales de la seguridad biológica del lugar. El artículo llama la atención sobre estas fallas y promueve una discusión sobre el protocolo de bioseguridad dentro de la acuicultura, abriendo el campo de mejora productiva con base en el mismo modelo que se aplica en otras áreas, como porcinocultura y avícola. De esta forma, la Escuela Agrícola de Jundiaí, unidad académica especializada en ciencias agrícolas, se garantiza una aplicación atemporal consistente en preceptos que pueden servir de modelo para cualquier actividad que implique la relación directa entre animales y humanos, asegurando una mayor estabilidad para que Los resultados deseados se logran sin la incidencia de agentes etiológicos y otros factores externos durante el proceso.

Palabras clave: Bioseguridad; Acuicultura; Control de Enfermedades; Agentes biologicos

\begin{abstract}
Animal production can suffer great impacts due to the diseases that affect it. Thus, in order to avoid the entry of pathogens in the production systems, the concept of biosecurity must be understood and applied continuously in all productive segments. This work aimed to evaluate the risk areas for pathogens entering the sectors of the aquaculture station at Jundiaí Agricultural School. Initially, a structured questionnaire with open questions about some functional aspects of the activities performed there was applied to the employees of the place. Their respective responses were stored in order to carry out a qualitative assessment, continuing the work of analysis and classification of management practices seen as fundamental to the maintenance of biosecurity. Thus, some structural and technical inadequacies that could affect vital points of the biological safety of the place were found. The article draws attention to these flaws and promotes a discussion regarding the biosecurity protocol within aquaculture, opening the scope for productive improvement based on the same model that is applied in other areas, such as pig farming and poultry farming. In this way, the Jundiaí Agricultural School, an academic unit specialized in agricultural sciences, is guaranteed a timeless application consisting of precepts that can serve as a model for any activity involving the direct relationship between animals and humans, ensuring greater stability so that desired results are achieved without the incidence of etiologic agents and other external factors during the process.
\end{abstract}

Keywords: Biosecurity; Aquaculture; Disease control; Biological agents; 


\section{INTRODUÇÃO}

O Brasil está entre os maiores produtores de produtos de origem animal do mundo, a produção de aves, suínos, bovinos, entre outras espécies é responsável por grande parte da nossa exportação. O consumo de produtos de origem animal no mercado interno, num país com mais de duzentos milhões de habitantes também tem uma enorme importância para sua população. Entre os pilares da produção animal está a sanidade, pois os impactos causados pelas doenças além de comprometerem economicamente os produtores, impacta nas relações de comércio interior e exterior, muitas vezes ocorrendo embargo das exportações quando as enfermidades estão listadas com obrigatoriedade de notificação as organizações de saúde animal internacionais.

Desta forma a ciência animal aprimora suas ações para evitar a entrada de patógenos nos sistemas de produção, assim a biosseguridade, um conceito usado a mais tempo pelo setor de produção avícola e suinícola deve ser implementado e inserido em todos os setores de produção animal inclusive na produção de organismos aquáticos. A aquicultura, sobretudo a criação de crustáceos, liderados pelos camarões são de extrema importância para a região nordeste do Brasil, maior produtora nacional. Enquanto escola para formação de profissionais das ciências agrárias, incluímos o setor de aquicultura na avaliação desses critérios que envolvem a biosseguridade, traçando assim o perfil da Estação de Aquicultura Dom Nivaldo Monte. 


\section{AVALIAÇÃO DE BIOSSEGURIDADE NA ESTAÇÃO DE AQUICULTURA FUNDAMENTAÇÃO TEÓRICA}

A aquicultura no Brasil, no período de 2005 a 2015, registrou um crescimento de $123 \%$ na produção nacional (EMBRAPA 2016). O setor da piscicultura é um dos que mais apresentam potencial de expansão, uma vez que a introdução de novas tecnologias na área tem ajudado a melhorar a disponibilidade hídrica, permitindo o pleno desenvolvimento da atividade em todas as regiões do país. Dados do Anuário Brasileiro da Piscicultura, publicado pela Associação Brasileira de Piscicultura em 2019, registraram um aumento de 4,5\% na produção nacional em 2018, valores que evidenciam o crescimento desse setor.

Como consequência desse avanço, a Aquicultura no Brasil atrai os olhares do mundo inteiro como oportunidade de negócios. Com isso, o país passou a receber não só investimentos de empresas nacionais, mas também de fortes grupos financeiros do exterior. E isto ocasionou um impacto direto no setor produtivo, que teve que se modernizar por completo para entrar nos padrões de qualidade e exigências internacionais, para que assim o nosso produto se fizesse presente nos grandes mercados do mundo.

Caminhando paralelamente com o desenvolvimento da aquicultura, os problemas com a mortalidade nas criações também aumentaram. Estima-se que, no Brasil, as enfermidades ocasionadas por patógenos sejam responsáveis por uma perda de mais de $15 \%$ da produção da piscicultura continental. Essa taxa, convertida para números, significa também uma perda de mais de U\$ 84 milhões de dólares nas receitas, ocasionadas diretamente e indiretamente pela ação desses agentes etiológicos (MARTINS e TAVARES-DIAS, 2017). Partindo dessa premissa, professores, técnicos e outros especialistas da área reforçam a necessidade de se adotar boas práticas de manejo sanitário na produção, defendendo a ideia de que não basta somente crescer a produção desordenadamente, mas também, e principalmente, é preciso prezar por todos os aspectos envolvidos na atividade, para que a aquicultura evolua em sua cadeia produtiva como um todo e se torne mais estável, amenizando assim as perdas ocasionadas por acometimento de doenças. (PÁDUA, 2017).

Em meio a essa necessidade de se adotar medidas que promovam uma produção eficiente e com menos riscos de ser afetada por uma enfermidade, o conceito de biosseguridade surge como um conjunto de estratégias e ações que atuam no combate a presença de patógenos em uma determinada produção (PATRÍCIO, 2004), apresentando-se como parte importante do desenvolvimento sustentável na aquicultura, garantindo que os organismos criados mantenhamse livres o quanto possível de doenças que venham a ocasionar perdas animais e prejuízos para o produtor. Estes conceitos baseiam-se no equilíbrio da produção, na qual a higiene, a organização e a economia caminham lado a lado, permitindo que todo e qualquer trabalho aquícola desempenhado em uma determinada área possua mais segurança para o consumidor. Com base nessas afirmações, podemos relacionar a biosseguridade não só com a aquicultura, mas também com outros segmentos de produção, uma vez que esses vários métodos preventivos são fundamentais para a estabilidade das condições sanitárias dos animais.

Considerando que projetos de pesquisa, extensão e ensino são executados na Estação de Aquicultura da Escola Agrícola de Jundiaí (EAJ), situada no município de Macaíba, região metropolitana de Natal, estado do Rio Grande do Norte, é fundamental serem observadas as normas de biosseguridade para garantir que os resultados destes trabalhos e de outras pesquisas científicas sejam os melhores possíveis, sem eventuais alterações por problemas com enfermidades. A execução de um plano de biosseguridade em qualquer setor de produção é importante para que toda uma estrutura de qualidade se sustente sem sofrer interferência de 
fatores externos. Para isso, é necessário possuir conhecimentos teóricos e práticos variados na área aquícola, bem como noções de manejo e outras técnicas da biosseguridade. 


\section{AVALIAÇÃO DE BIOSSEGURIDADE NA ESTAÇÃO DE AQUICULTURA}

\section{METODOLOGIA}

Este trabalho é um estudo de caso que fez o mapeamento relacionado a biosseguridade, foi realizado na estação de aquicultura Dom Nivaldo Monte da Escola Agrícola de Jundiaí durante o ano de 2017. Foi seguido o protocolo de identificação de áreas biosseguras propostos por (LUZ, G. E., 2016). As áreas avaliadas na estação foram: Laboratório de qualidade de água, Laboratório de carcinicultura, Laboratório de piscicultura ornamental e Laboratório de larvicultura.

A primeira etapa do trabalho foi o levantamento de informações geográficas. Com auxílio do programa 'Sketchup', a planta da Estação de Aquicultura Sebastião Monte, da Escola Agrícola de Jundiaí, foi desenvolvida de modo a permitir uma análise minuciosa de todos os locais de produção, compreendendo também as relações de espaço existentes entre cada área e como os fatores externos poderiam estar presentes no meio.

Figura 1 - Planta da estação de Aquicultura Sebastião Monte - EAJ/UFRN

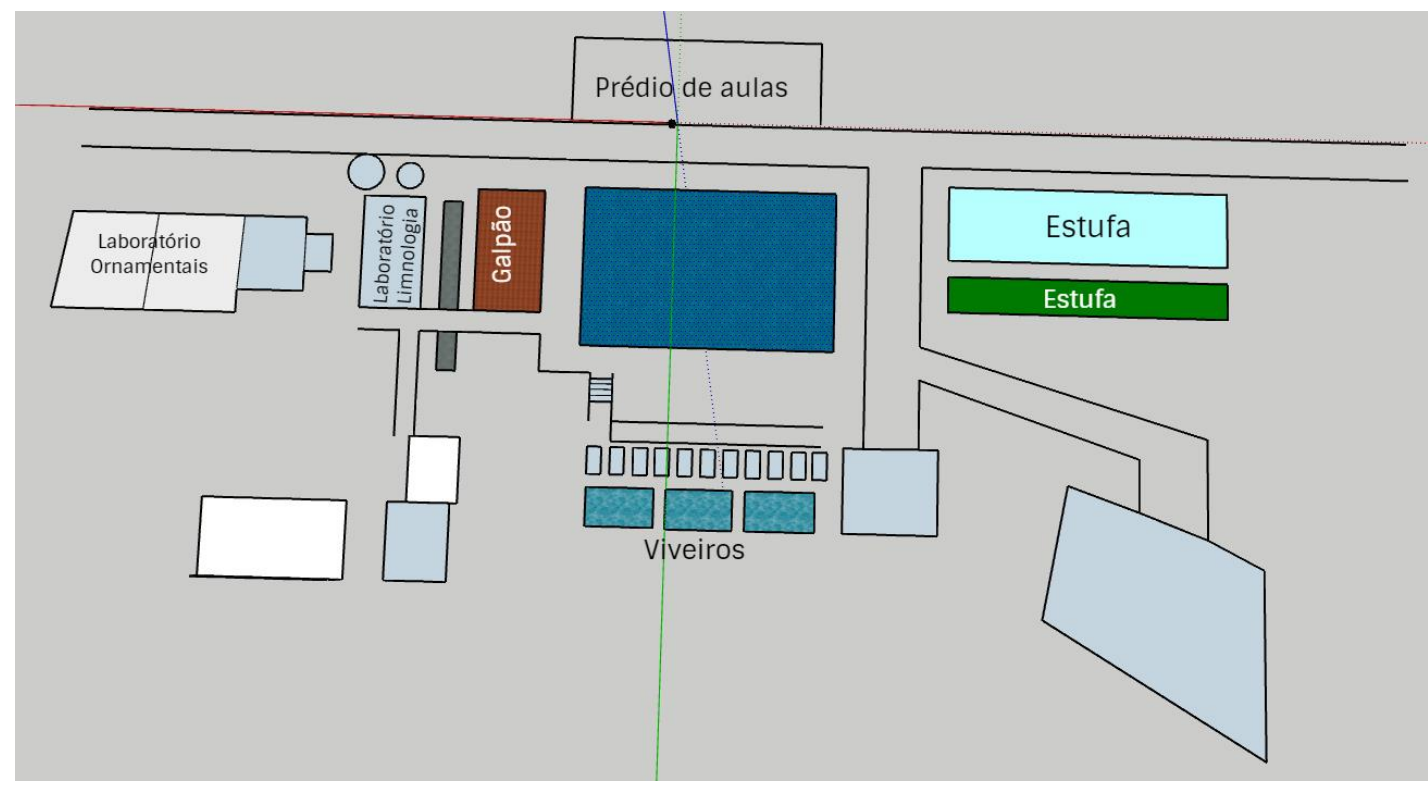

(Fonte: Arquivo desenvolvido pelos autores - 2017)

A segunda fase do trabalho consistiu na aplicação de um questionário aos colaboradores (técnicos, terceirizados e estagiários) a fim de se obter informações detalhadas quanto ao funcionamento dos locais. A avaliação continha 10 perguntas abertas relacionadas a Biosseguridade.

1. Controle de pessoas - É fundamental que todo setor de produção tenha o controle de quem pode ou não adentrar nas instalações. Essa medida ajuda a impedir o ingresso de agentes etiológicos que eventualmente poderiam acometer todo o sistema de produção.

2. Higienização pessoal - A preocupação com as condições sanitárias deve ter como prioridade a higienização daqueles que estão trabalhando diretamente com os animais de cada setor. Dessa forma, é preciso que hajam áreas disponíveis para essa descontaminação dos funcionários antes e depois de realizarem atividades no local. 
3. Unidade de quarentena - A quarentena é o período em que um determinado organismo vivo permanece em isolamento com o propósito de identificar as suas condições de saúde antes de inseri-lo em um novo ambiente. Dentro da Aquicultura, a técnica é aplicada com o propósito de proteger os animais de um determinado cultivo da ação de agentes infecciosos.

4. Controles de vetores e animais sinantrópicos - Os roedores podem ser um problema em qualquer produção por trazerem danos não só a estrutura, mas também por transmitirem mais de 32 doenças a homens e animais. O controle desse problema pode ser feito através do uso de recursos químicos ou de armadilhas mecânicas (BARCELLOS et al., 2008)

5. Descarte dos animais mortos - Os animais que são acometidos pelos agentes etiológicos e acabam morrendo causam preocupação para todo produtor. $\mathrm{O}$ descarte correto dessas carcaças evitam não só que a contaminação se espalhe para os organismos saudáveis, mas também a poluição ambiental.

6. Descarte da água - Em qualquer segmento de produção, a água exerce um papel de protagonismo. Na Aquicultura, esse destaque é ainda maior, já que tudo está relacionado a ela. Dessa forma, a preocupação com o destino e a qualidade da mesma é uma forma de garantir o bem-estar dos animais cultivados e de todo o ambiente ao redor. Além disso, a incidência de agentes infecciosos na água é muito grande, uma vez que em condições ideais de temperatura e concentração de materiais orgânicos, as bactérias podem se multiplicar facilmente (BARCELLOS et al., 1995). Já a umidade é um fator importante no ciclo de vida dos protozoários (FRASER et al., 1991).

7. Sanitização dos equipamentos de manejo - Os equipamentos utilizados durante a criação precisam passar sempre pelo processo de esterilização. Essa precaução deve ser adotada a fim de evitar que algumas bactérias se alojem nos materiais e acabem atuando como propagadores de doenças.

8. Equipamentos de proteção para funcionários - Para garantir que todo o processo de Biosseguridade seja bem desempenhado, é preciso também envolver práticas de Biossegurança dentro das atividades. Desse modo, o cuidado com a proteção daqueles que atuam diretamente no cultivo é vital para o sucesso do mesmo. Botas, luvas e jalecos são acessórios obrigatórios para qualquer técnico/produtor.

9. Vazio sanitário - Uma das práticas mais importantes da Biosseguridade é o 'vazio sanitário'. Nela, as instalações onde ocorrem o processo produtivo são submetidas à limpeza e desinfecção total, e em seguida ficam fechadas por um determinado período (geralmente 24-48 horas).

10. Uso de produtos químicos - A presença dos produtos químicos em qualquer cultivo é fundamental, uma vez que eles exercem o papel de protagonistas no combate aos agentes etiológicos. 


\section{AVALIAÇÃO DE BIOSSEGURIDADE NA ESTAÇÃO DE AQUICULTURA}

A fim de aumentar a precisão nas respostas obtidas, também foram adotados alguns critérios técnicos de seleção dos entrevistados, tais como:

1. Tempo de experiência no setor;

2. Função desempenhada;

3. Grau de formação (técnico, graduado ou funcionário terceirizado)

Para concluir a etapa de levantamento de informações, as respostas colhidas no questionário foram organizadas em uma planilha do Excel para ser feita a caracterização dos resultados.

Em posse dos dados necessários para o desenvolvimento do projeto, deu-se início a etapa de caracterização dos resultados. A metodologia utilizada para a análise qualitativa foi fundamentada no trabalho de (GARCIA, S. K e GONÇALVES, J. P. M, 2012) e composta considerando critérios importantes para a manutenção das práticas realizadas na estação de Aquicultura da EAJ. Estas não levam em conta apenas aspectos técnicos e agentes etiológicos em si, mas também seres humanos e animais, complexos em suas naturezas e relações. Dessa forma, algumas adaptações foram feitas para que o resultado final fosse o mais próximo possível da realidade vivenciada no local.

1. A pontuação máxima que um setor poderia receber no questionário era de 100 (cem) e a mínima de 0 (zero). Ao final, essas notas seriam somadas e divididas por 10 a fim de se obter a média aritmética.

2. O Laboratório de Limnologia não se encaixava em determinados parâmetros avaliados pelo questionário. Dessa forma, sua pontuação foi ajustada proporcionalmente ao número de técnicas que o setor estava apto a realizar.

3. Avaliação por conceitos: para cada técnica de Biosseguridade avaliada no questionário, foram atribuídas 3 pontuações distintas:

a. 10 pontos - Caso o setor desempenhe a técnica corretamente e garanta um risco baixo para a produção.

b. 5 pontos - Caso o setor realize a técnica, porém não de forma totalmente correta, acarretando um risco moderado.

C. 0 pontos - Caso o setor não realize a técnica e apresente alto risco para a produção.

Os resultados obtidos e suas respectivas classificações serão apresentados a seguir, na seção de resultados e discussão. 


\section{RESULTADOS E DISCUSSÃO}

Tabela 1 - Respostas do questionário de Biosseguridade e suas respectivas classificações de risco para cada setor

\begin{tabular}{|c|c|c|c|c|c|}
\hline Práticas de Biosseguridade / Locais & Estufa (Carcinicultura) & $\begin{array}{c}\text { Galpão de Larvicultura } \\
\text { (Piscicultura) }\end{array}$ & $\begin{array}{l}\text { Viveiros do Deckel } \\
\text { (Piscicultura) }\end{array}$ & $\begin{array}{l}\text { Laboratório de } \\
\text { Limnologia }\end{array}$ & $\begin{array}{c}\text { Laboratório de } \\
\text { Ornamentais }\end{array}$ \\
\hline Controle de pessoas (entrada) & Não possui & $\begin{array}{c}\text { Somente estagiários e } \\
\text { pessoas autorizadas }\end{array}$ & Não possui & $\begin{array}{c}\text { Somente estagiários e } \\
\text { pessoas autorizadas }\end{array}$ & $\begin{array}{c}\text { Somente estagiários e } \\
\text { pessoas autorizadas }\end{array}$ \\
\hline Área de higienização pessoal & Não possui & Não posssui & Não possui & Possui & Não possui \\
\hline $\begin{array}{l}\text { Unidade de quarentena para animais } \\
\text { recém-chegados }\end{array}$ & Possui & Não posssui & Não possui & - & Possui \\
\hline $\begin{array}{c}\text { Controle de vetores e animais } \\
\text { sinantrópicos }\end{array}$ & Não possui & Possui & Não possui & Não possui & Não possui \\
\hline Descarte dos animais que morrem & Sem padrão & $\begin{array}{l}\text { Enterra-se fora do } \\
\text { galpão }\end{array}$ & $\begin{array}{l}\text { Reutilizados na } \\
\text { alimentação de outros } \\
\text { organismos }\end{array}$ & - & Sem padrão \\
\hline Descarte da água utilizada no local & $\begin{array}{l}\text { Através da rede do } \\
\text { setor }\end{array}$ & $\begin{array}{l}\text { Vai pro sistema de } \\
\text { recirculação }\end{array}$ & $\begin{array}{l}\text { Vai pro sistema de } \\
\text { recirculação }\end{array}$ & - & $\begin{array}{l}\text { Através da rede do } \\
\text { setor }\end{array}$ \\
\hline $\begin{array}{l}\text { Realiza sanitização dos equipamentos } \\
\text { de manejo }\end{array}$ & Sim & Sim & Não & Sim & Sim \\
\hline $\begin{array}{l}\text { Possui equipamentos de proteção para } \\
\text { os funcionários }\end{array}$ & Não possui & Possui & Possui & Possui & Possui \\
\hline Realiza a prática do 'vazio sanitário' & Não & Não & Não & Não & Não \\
\hline $\begin{array}{l}\text { Utilizam produtos químicos durante a } \\
\text { criação }\end{array}$ & Sim & Sim & Sim & Sim & Sim \\
\hline LEGENDAS: $\begin{array}{c}\text { O Risco } \\
\text { O Risco } \\
\text { O Risco }\end{array}$ & $\begin{array}{l}\text { Ito } \\
\text { noderado } \\
\text { aixo }\end{array}$ & O Técnica & $\begin{array}{l}\text { de Biossegurida } \\
\text { ões }\end{array}$ & & \\
\hline
\end{tabular}

(Fonte: Arquivo desenvolvido pelos autores - 2020) 


\section{AVALIAÇÃO DE BIOSSEGURIDADE NA ESTAÇÃO DE AQUICULTURA}

Gráfico 1 - Classificação de cada setor (com base na tabela 1) pelo número de técnicas de Biosseguridade presentes na produção

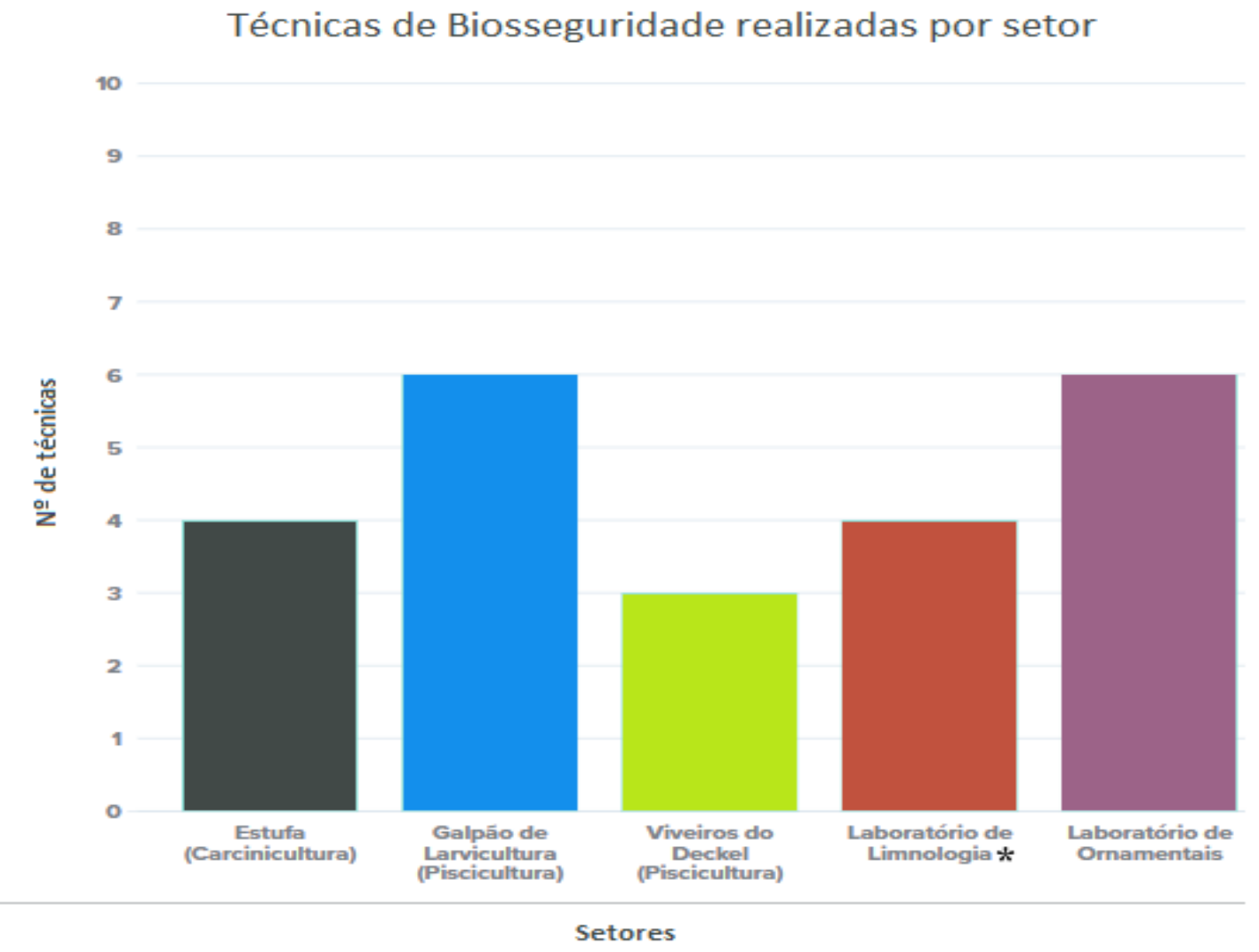

(Fonte: Arquivo desenvolvido pelos autores - 2020)

De acordo com o gráfico 1, os laboratórios de peixes ornamentais e o de larvicultura foram os que apresentaram o maior número de técnicas de biosseguridade desempenhadas (6/10), enquanto que os viveiros do deckel (3/10) obtiveram o pior desempenho. Estes resultados estão dentro das expectativas iniciais do trabalho, haja visto que a abordagem do tema dentro de algumas áreas de produção não ocorre de forma ampla, resultando no desconhecimento das boas práticas sanitárias por parte de alguns funcionários.

É importante também chamar a atenção para a prática de 'vazio sanitário', que aparece no questionário como sendo a $9^{\circ}$ pergunta (tabela 1), e que não é desempenhada por nenhum dos 5 setores avaliados. Segundo (OLIVEIRA, 2010), a realização dessa técnica de caráter simples colabora diretamente com a melhoria da saúde animal e do produto final que se deseja obter. 
Tabela 2 - Pontuação no questionário e média final de cada setor avaliado

\begin{tabular}{|c|c|c|}
\hline \multicolumn{1}{|c|}{ INSTALAÇÕES } & $\begin{array}{c}\text { PONTUAÇÃO NO } \\
\text { QUESTIONÁRIO }\end{array}$ & MÉDIA FINAL \\
\hline Estufa & $35 / 100$ & 3,5 \\
\hline Galpão de Larvicultura & $65 / 100$ & 6,5 \\
\hline Viveiros Deckel & $25 / 100$ & 2,5 \\
\hline Laboratório de Limnologia & $45 / 70$ & 6,4 \\
\hline Laboratório de & $55 / 100$ & 5,5 \\
\hline Ornamentais & & \\
\hline
\end{tabular}

(Fonte: Arquivo desenvolvido pelos autores - 2020)

Aplicando o sistema de avaliação previamente descrito na metodologia, foram obtidas as pontuações de cada setor nos questionários. A utilização da média aritmética como forma de definição dos valores finais permitiu que fossem feitas correções em relação ao Laboratório de Limnologia, que por não se encaixar em 3 das 10 perguntas realizadas na primeira etapa, seria prejudicado de forma direta na avaliação do seu desempenho, ficando com uma pontuação inferior em relação aos outros. Este ajuste assegurou uma nota precisa ao local (a $2^{\circ}$ maior pontuação entre setores).

Tabela 3 - Classificação de risco de cada setor

\begin{tabular}{|c|c|}
\hline SETOR & GRAU DE RISCO \\
\hline Estufa & Classe 2 \\
\hline Galpão Larvicultura & Classe 1 \\
\hline Viveiros do Deckel & Classe 3 \\
\hline Laboratório de Limnologia & Classe $\mathbf{1}$ \\
\hline Laboratório de Ornamentais & Classe 2 \\
\hline
\end{tabular}

(Fonte: Arquivo desenvolvido pelos autores - 2020)

Diante dos resultados obtidos, a classificação do grau de risco dos setores mostrou que a maioria dos setores da estação de aquicultura da Escola Agrícola de Jundiaí se encontram em níveis aceitáveis em relação aos procedimentos técnicos que integram a biosseguridade. É possível ainda constatar que as notas mais baixas de avaliação, Viveiros do Deckel (nota 2,5) e Estufas de Carcinicultura (nota 3,5), são de áreas mais isoladas geograficamente em relação aos outros três setores mais bem avaliados. Isto pode ser confirmado com base na planta da estação de aquicultura (figura 1). 


\section{AVALIAÇÃO DE BIOSSEGURIDADE NA ESTAÇÃO DE AQUICULTURA}

\section{CONCLUSÕES}

A partir da observação dos resultados, é possível afirmar que alguns preceitos de higiene estão sendo seguidos adequadamente nos setores, uma vez que 4 das 5 instalações avaliadas apresentaram classificação de risco entre 1 e 2, que representam grau baixo e moderado, respectivamente.

Outro ponto a ser destacado é o desconhecimento em relação aos protocolos de biosseguridade por parte de alguns dos entrevistados. Isto permite a conclusão de que o tema é pouco abordado não só dentro da aquicultura, mas também em outros setores produtivos. A falta de conhecimento básico de medidas de segurança biológica pode colocar em risco todo um trabalho, gerando prejuízos financeiros incalculáveis.

O propósito deste trabalho era apresentar um panorama geral dos setores da Aquicultura em relação aos protocolos de biosseguridade. Ao apontar as falhas e os acertos de cada instalação, abre-se espaço para o aprimoramento produtivo, que está diretamente relacionado com um melhor gerenciamento das atividades desenvolvidas no local. Uma vez que corrigidas essas falhas, o papel de protagonismo da Escola Agrícola de Jundiaí como unidade acadêmica de pesquisa agrária é reconhecido pelos exigentes padrões do mercado. 


\section{REFERÊNCIAS}

ANDREATTI FILHO, R. L.; PATRÍCIO, I. S. Biosseguridade da granja de frangos de corte. Campinas: FACTA, p. 169-177, 2004.

Aquicultura brasileira cresce $123 \% \mathrm{em} \mathrm{dez}$ anos. Empresa Brasileira de Pesquisa Agropecuária - EMBRAPA. Brasília, 13 de dez. de 2016. Disponível em: <https://www.embrapa.br/busca-de-noticias/-/noticia/18797150/aquicultura-brasileira-cresce123-em-dez-anos>. Acesso em: 12 de set. de 2020.

BARCELLOS, D. E. S. N. Influência da qualidade da água sobre alguns parâmetros da produtividade e sanidade em suinocultura. In: VII Congresso Brasileiro de Veterinários Especialistas em Suínos. 1995.

BARCELlOS, D. E. S. N.; MORES, T. J.; SANTI, M.; GHELLER, N. B. Avanços em programas de biosseguridade para a suinocultura. Acta Scientiae Veterinariae. Porto Alegre, v. 36, 2008.

FRASER, D.; PATIENCE, J. F.; MCLEESE, J. M. Water for piglets and lacting sows quantity, quality and quandaries. In: Recents Advances in Animal Nutrition. p. 137-160. 1991.

GARCIA, S. K e GONÇALVES, J. P. M. Suinocultura mineira e sua defesa sanitária. Revista V\&Z em Minas. Ano XXII, Ed. 114. P. 44-51, 2012.

História da Escola Agrícola de Jundiaí. EAJ-UFRN. Macaíba, 2011. Disponível em: $<$ http://www.eaj.ufrn.br/site/pagina.php?a=int_historico>.

LUZ, G. E.; PEDROSO, A. C. Avaliação das práticas de biosseguridade em granjas de frango de corte no município de Realeza-PR. UFFS, 2016.

MARTINS, M. L; TAVARES-DIAS, M. An overall estimation of losses caused by diseases in the Brazilian fish farm. Embrapa Amapá, 2017. Disponível em: < https://ainfo.cnptia.embrapa.br/digital/bitstream/item/165759/1/CPAF-AP-2017-An-overallestimation-of-losses.pdf>. Acesso em: 26 de Maio de 2020.

OLIVEIRA, J.R. et al. Biossegurança e vazio sanitário das instalações zootécnicas. PUBVET, Londrina, V. 4, N. 7, Ed. 112, Art. 754, 2010.

PÁDUA, de B. S. A Biosseguridade é a solução para os problemas sanitários da Piscicultura brasileira?. Aquaculture Brasil, 2017.

The state of world fisheries and aquaculture: opportunities and challenges. FAO. Rome, 2014. 